\title{
REGULAR SKEW GROUP RINGS
}

\author{
RICARDO ALFARO, PERE ARA and ANGEL DEL RÍo
}

(Received 1 February 1992; revised 6 May 1992)

Communicated by P. Schultz

Dedicated to the memory of Pere Menal

\begin{abstract}
Let $G$ be a group acting on a ring $R$. We study the problem of determining necessary and sufficient conditions in order that the skew group ring $R G$ be von Neumann regular. Complete characterizations are given in some particular situations, including the case where all idempotents of $R$ are central. For a regular ring $R$ admitting a $G$-invariant pseudo-rank function $N$, with $G$ finite, we obtain a necessary condition for $R G$ being regular in terms of the induced action of $G$ on the $N$-completion of $R$.
\end{abstract}

1991 Mathematics subject classification (Amer. Math. Soc.): primary 16S35; secondary 23B45.

\section{Introduction}

Associated to an action of a group $G$ on a ring $R$, there are two rings: the fixed ring $R^{G}$ and the skew group ring $R G$ (see Section 1 for definitions). The relationship between the structure of these three rings, $R, R^{G}$ and $R G$ has been intensively studied by many authors, see $[11,17,19]$ for instance.

In this paper we study when the skew group ring is regular in the sense of von Neumann. When the action is trivial the skew group ring is just the classical group ring $R[G]$, and it is well known that $R[G]$ is regular if and only if $R$ is regular, $G$ is locally finite and the order of any finite subgroup of $G$ is invertible in $R$; this result is due to contributions of Auslander [2], Connell [5] and Villamayor [21].

The first author was partially supported by a grant from the Faculty Development Fund of the University of Michigan-Flint and a fellowship from the C. R. M. Bellaterra, Spain. The second author was partially supported by CICYT grant PB89-0296 and the third author was partially supported by CICYT grant PB87-0703.

(C) 1995 Australian Mathematical Society $0263-6115 / 95 \$ A 2.00+0.00$ 
In the history of the study of skew group rings (or more generally crossed products) there is Maschke's standard argument which has been extensively used when $G$ is finite and the order of $G$ is invertible in $R$. This argument also works when the trace of the center of $R$ contains the unit of $R$, and allows us to obtain a sufficient condition for $R G$ to be regular. This extension was inspired by some results on separable functors; see $[13,20]$. In the first section we prove this extension and give general notation and definitions.

In the second section we obtain some necessary conditions for $R G$ to be regular. Namely we prove that if $R G$ is regular, then $R$ is regular, $G$ is locally finite and for every finite subgroup $H$ of $G, 1 \in \operatorname{tr}_{H}(R)$ and $R^{H}$ is regular. As a consequence, the general problem can be reduced to the particular case of finite groups. In some particular cases ( $R$ commutative or $G$-Galois actions) these necessary conditions are also sufficient.

In the third section we study the particular case when $R$ is abelian regular (that is, regular with all idempotents central). In this case the necessary conditions turn out to be sufficient. It is worth noting that $R^{G}$ is always abelian regular if $R$ is abelian regular (because every element of an abelian regular ring has a (unique) group inverse).

In the fourth section we start by giving necessary and sufficient conditions for $R G$ to be regular when $R$ is regular right self-injective and $G$ is finite. We show that regular, semiprimitive and semiprime are equivalent conditions for the skew group ring $R G$. In this particular case, Passman's results on semiprime crossed products are useful. Finally we deal with pseudo-rank functions, obtaining that if $N$ is a $G$ invariant pseudo-rank function then there is a natural pseudo-rank function $\tilde{N}$ on $R G$ and the action of $G$ on $R$ can be extended to its completion $\bar{R}$ under the topology associated to $N$. Moreover, the completion of $R G$ under $\tilde{N}$ is homeomorphic under a ring isomorphism to $\bar{R} G$. This result allows us to give an example of a simple regular ring $R$ with a finite group $G$ of outer automorphisms such that $R G$ and $R^{G}$ are not regular.

\section{Sufficient Conditions}

Throughout $R$ will denote an associative ring with unity, $Z(R)$ will denote the center of the ring $R$, and $G$ will stand for a group action on $R$, that is, there is a group homomorphism $\sigma: G \rightarrow$ Aut $(R)$. If $r \in R$ and $g \in G$, then ${ }^{g} r$ will stand for $\sigma_{g}(r)$.

The skew group ring $R G$ is a free left $R$-module with basis $G$ (that is, the elements of $R G$ are finite linear combinations $\sum_{g \in G} r_{g} g$, with $r_{g} \in R$ and $g \in G$ ) and product given by $(r g)(s h)=r\left({ }^{g} s\right) g h$. The fixed subring of $R$ under $G$ is $R^{G}=\{r \in$ $R \mid{ }^{g} r=r$, for all $\left.g \in G\right\}$. If $G$ is finite, the trace is the map $\operatorname{tr}_{G}: R \rightarrow R^{G}$, with $\operatorname{tr}_{G}(r)=\sum_{g \in G}{ }^{g_{r}}$. Note that if $H$ is a subgroup of $G$ and $X$ is a $G$-invariant subset 
of $R$, then $\operatorname{tr}_{G}(X) \subseteq \operatorname{tr}_{H}(X)$. Indeed, if $x \in X$ and $A$ is a right transversal of $H$, then $\operatorname{tr}_{G}(x)=\operatorname{tr}_{H}\left(\sum_{a \in A}{ }^{a} X\right)$.

The main interest of this paper is to obtain necessary and sufficient conditions for $R G$ to be regular. The case when $G$ acts trivially on $R$ was solved by Auslander [2], Connell [5] and Villamayor [21]: the group ring $R[G]$ is regular if and only if $R$ is regular, $G$ is locally finite and the order of every finite subgroup of $G$ is invertible in $R$. In the general case it is well known that the sufficient conditions remain true.

Proposition 1.1 (see [19, Prop. 17.2]). If $R$ is regular, $G$ is locally finite and the order of any finite subgroup is invertible in $R$ then $R G$ is regular. The same is true when we consider a crossed product $R * G$.

The converse does not hold as the following example shows:

EXAMPLE 1.2. Let $A$ be a regular ring, $n \geq 1$, and set $R=A^{n}$. Let $\sigma$ be an automorphism of $R$ given by a cyclic permutation of order $n$ on the factors of $R$. Put $G=\langle\sigma\rangle$. Then $R G$ is regular (see Theorem 1.3 below) but $|G|=n$ is invertible in $R$ if and only if it is invertible in $A$.

Proposition 1.1 can be improved using Maschke's standard argument as follows:

THEOREM 1.3. Let $G$ be a locally finite group acting on a regular ring $R$. Assume that for every finite subgroup $H$ of $G, 1 \in \operatorname{tr}_{H}(Z(R))$. Then $R G$ is regular.

PROOF. It is clear that it is enough to prove the theorem for $G$ finite with $1 \epsilon$ $\operatorname{tr}_{G}(Z(R))$. Let $r \in Z(R)$ be such that $\operatorname{tr}_{G}(r)=1$. Let $I$ be a principal left ideal of $R G$. Then $I$ is a direct summand of $R G$ as left $R$-modules. Let $\rho \in \operatorname{Hom}_{R}(R G, I)$ be a projection. Then $f(m)=\sum_{g \in G} g r \rho\left(g^{-1} m\right)$ is a projection and $f \in \operatorname{Hom}_{R G}(R G, I)$. It follows that $R G$ is a regular ring.

In Section 3 we will give an example of a regular skew group ring $R G$ with $G$ finite such that 1 does not belong to $\operatorname{tr}(Z(R))$.

\section{Necessary conditions}

In this section we give some necessary conditions for $R G$ to be regular. We then prove that in some particular cases these conditions are also sufficient, and also give some examples which show that these necessary conditions are not sufficient in general.

Let $G$ be a group acting on a ring $R$. We can consider $R$ as a right $R^{G}$-module by restriction of scalars and as a left $R G$-module by the rule $(r g) \cdot s=r\left({ }^{8} s\right)$ for $r, s \in R$, 
$g \in G$; so $R$ is an $\left(R G, R^{G}\right)$-bimodule. Let $A: R G \rightarrow R$ be the canonical bimodule homomorphism given by $A\left(\sum_{g \in G} a_{g} g\right)=\sum_{g \in G} a_{g}$. When $G$ is finite $\pi$ will denote the element $\sum_{g \in G} g$ in $R G$.

The following lemmas are well known:

LEMMA 2.1. Assume $G$ is finite. For any $\alpha \in R G, \alpha \pi=A(\alpha) \pi$.

LEMMA 2.2. Let $X$ be a set of generators of $G$. Then $\{x-1 \mid x \in X\}$ is a set of generators of $\operatorname{Ker}(A)$ as a left $R G$-module.

PROOF. If $\sum_{g \in G} a_{g} g \in \operatorname{Ker}(A)$, then $\sum_{g \in G} a_{g} g=\sum_{g \in G} a_{g} g-\sum_{g \in G} a_{g}=$ $\sum_{g \in G} a_{g}(g-1)$. Thus $\{g-1 \mid g \in G\}$ generates $\operatorname{Ker}(A)$. Consider now $(g-1)$ as an element of $R^{G} G=R^{G}[G]$. Then, by [18, Lemma 3.1.1], $\{x-1 \mid x \in X\}$ generates the augmentation ideal of $R^{G}[G]$ and hence $(g-1) \in \sum_{x \in X} R^{G}(x-1)$ for all $g \in G$.

LEMMA 2.3 ([11, Prop. 0.3]). $R^{G}$ is canonically isomorphic to End $\left({ }_{R G} R\right)$.

Now we can give the necessary conditions:

THEOREM 2.4. Let $G$ be a group acting on a ring $R$. Assume that $R G$ is regular. Then the following conditions hold:

(a) For every subgroup $H$ of $G, R H$ is regular. In particular $R$ is regular.

(b) $G$ is locally finite.

(c) For every finite subgroup $H$ of $G, 1 \in \operatorname{tr}_{H}(R)$.

(d) For every finite subgroup $H$ of $G, R^{H}$ is regular.

ProOF. (a) is trivial. By (a) we can assume that $G$ is finitely generated. By Lemma $2.2, \operatorname{Ker}(A)$ is finitely generated; therefore ${ }_{R G} R \cong R G / \operatorname{Ker}(A)$ is finitely presented and hence projective. Let $A^{\prime}: R \rightarrow R G$ be the split homomorphism of $A$. Set $A^{\prime}(1)=\sum_{h \in G} a_{h} h$. Then for any $g \in G$,

$$
\sum_{h \in G} a_{h} h=A^{\prime}(1)=A^{\prime}(g .1)=g A^{\prime}(1)=g \sum_{h \in G} a_{h} h=\sum_{h \in G}{ }^{g}\left(a_{h}\right) g h .
$$

Thus $a_{g h}={ }^{g}\left(a_{h}\right)$ for all $g, h \in G$ and hence $a_{g} \neq 0$ for any $g \in G$. We conclude that $G$ is finite and this proves (b). Moreover, $1=A A^{\prime}(1)=\operatorname{tr}\left(a_{e}\right)$, where $e$ is the unit of $G$, so (c) follows. Finally, since ${ }_{R G} R$ is finitely generated projective and $R G$ is regular, $R^{G} \cong$ End $\left({ }_{R G} R\right)$ is regular.

By Theorem 2.4 the problem of studying when $R G$ is regular can be reduced to the case when $G$ is finite. Moreover the case when $R$ is commutative is completely solved by Theorems 1.3 and 2.4. Explicitly: 
COROLlaRY 2.5. Assume $R$ is commutative. $R G$ is regular if and only if $R$ is regular, $G$ is locally finite and $1 \in \operatorname{tr}_{H}(R)$ for every finite subgroup $H$ of $G$.

REMARK. When $R$ is not commutative, a similar result is not possible. Indeed, let $G$ be a finite group and let $A$ be a gr-regular $G$-graded ring (that is, every graded left $A$-module is flat, see [14]); also assume $A$ is not regular. (For example a group ring $S[G]$ with $S$ regular, $G$ finite and $|G|$ not invertible in $S$.) Then $G$ acts on the smash product $R=A \# G$, and $R G \cong M_{|G|}(A)$ is not regular although $R$ is regular and $1=\operatorname{tr}\left(p_{1}\right)([4,3])$.

Theorem 2.4 also has some consequences for the regularity of crossed products. A crossed product $R * G$ is a $G$-graded ring $A$ (that is, a ring with a decomposition into additive subgroups $A=\bigoplus_{g \in G} A_{g}$ such that $A_{g} A_{h} \subseteq A_{g h}$, for every $g, h \in G$ ), in which $A_{e}=R$ and $A_{g}$ contains an invertible element for every $g \in G$.

COROLLARY 2.6. If $R * G$ is a regular crossed product, then $G$ is torsion.

PrOOF. For any subgroup $H$ of $G, R * H$ is also regular, therefore it is enough to prove that if $G$ is cyclic, then it is finite. For that we use the fact that any crossed product on a cyclic group can be expressed as a skew group ring using a diagonal change of basis. Now, by Theorem 2.4 (b), $G$ is cyclic and locally finite, and hence finite.

REMARK. It would be interesting to know if in regular crossed products the group must be locally finite.

From Theorem 2.4 we have that if $R G$ is regular and $G$ is finite, then $R^{G}$ is regular. But if $G$ is not finite, the regularity of $R G$ does not imply the regularity of $R^{G}$, as the following example shows:

EXAMPLE 2.7. Let $K$ be a field of characteristic $0, S=M_{2}(K)$ and $P=\left(\begin{array}{rr}1 & 0 \\ -1 & 1\end{array}\right)$. Consider $\alpha$, the inner automorphism of $S$ defined by $P$. Let $\mathbb{N}$ denote the set of natural numbers; for any element $x \in S^{\mathbb{N}}$ and any $n \in \mathbb{N}, x_{n}$ will denote the $n$-th entry of $x$. Let $R$ be the following subring of the product ring $S^{N}$ :

$$
R=\left\{x \in S^{\mathbb{N}} \mid \text { there exists } n \in \mathbb{N} \text { such that } x_{n}=x_{n+k} \text { for all } k \in \mathbb{N}\right\} .
$$

For every $n \in \mathbb{N}$ consider the automorphism of $R$ given by:

$$
\left(\sigma_{n}(x)\right)_{m}= \begin{cases}\alpha^{-1}\left(x_{m-1}\right) & \text { if } 1<m \leq 2 n \text { and } m \text { is even, } \\ \alpha\left(x_{m-1}\right) & \text { if } 1<m<2 n \text { and } m \text { is odd, } \\ \alpha\left(x_{2 n}\right) & \text { if } m=1, \\ x_{m} & \text { if } 2 n<m .\end{cases}
$$


If any element of $S^{\mathbb{N}}$ is represented by a column vector with matrix entries, then $\sigma_{n}$ can be represented by the $\mathbb{N} \times \mathbb{N}$ matrix:

$$
A_{n}=\left(\begin{array}{cccccc|c}
0 & 0 & 0 & \ldots & 0 & \alpha & \\
\alpha^{-1} & 0 & 0 & \ldots & 0 & 0 & \\
0 & \alpha & 0 & \ldots & 0 & 0 & \mathrm{O} \\
0 & 0 & \alpha^{-1} & \ldots & 0 & 0 & \\
. & . & . & \ldots & . & . & \\
. & . & . & \ldots & . & . & \\
0 & 0 & 0 & \ldots & \alpha^{-1} & 0 & \\
\hline \multicolumn{7}{c}{0} \\
\hline
\end{array}\right.
$$

Indeed $\sigma_{n}(x)=A_{n} x$ for any $x \in S^{\mathbb{N}}$. Let $G$ be the subgroup of Aut $(R)$ generated by $\left\{\sigma_{n} \mid n \in \mathbb{N}\right\}$.

$G$ is locally finite. To prove this it is enough to show that for every $n \in \mathbb{N}$, $\sigma_{1}, \sigma_{2}, \ldots, \sigma_{n}$ generates a finite group. Let $k_{1}, k_{2}, \ldots, k_{p} \in\{1,2, \ldots, n\}$. Then $\left(\sigma_{k_{p}} \cdots \sigma_{k_{2}} \sigma_{k_{1}}(x)\right)_{m}=\beta_{p}\left(\sigma_{k_{p-1}} \cdots \sigma_{k_{2}} \sigma_{k_{1}}(x)\right)_{r_{p}}$ where

$$
\left\{\begin{array}{lll}
r_{p}=m & \text { and } \beta_{p}=1 & \text { if } 2 k_{p}<m, \\
r_{p}=m-1 & \text { and } \beta_{p}=\alpha^{(-1)^{m+1}} & \text { if } 1 \neq m \leq 2 k_{p}, \\
r_{p}=2 k_{p} & \text { and } \beta_{p}=\alpha & \text { if } m=1 .
\end{array}\right.
$$

Thus $\left(\sigma_{k_{p}} \cdots \sigma_{k_{2}} \sigma_{k_{1}}(x)\right)_{m}=\beta_{p} \cdots \beta_{2} \beta_{1}\left(x_{r_{1}}\right)$, where

$$
\left\{\begin{array}{lll}
r_{i}=r_{i+1} & \text { and } \beta_{i}=1 & \text { if } 2 k_{i}<r_{i+1}, \\
r_{i}=r_{i+1}-1 & \text { and } \beta_{i}=\alpha^{(-1)^{r_{i+1}+1}} & \text { if } 1 \neq r_{i+1} \leq 2 k_{i}, \\
r_{i}=2 k_{i} & \text { and } \beta_{i}=\alpha & \text { if } r_{i+1}=1 .
\end{array}\right.
$$

If $\beta_{i}$ and $\beta_{i+1}$ are not 1 , then they are inverse to each other because in this case $r_{i}$ and $r_{i+1}$ have different parity and therefore $\beta_{p} \cdots \beta_{2} \beta_{1}$ is equal to either $1, \alpha$ or $\alpha^{-1}$. It is now clear that $\sigma_{k_{p}} \cdots \sigma_{k_{2}} \sigma_{k_{1}}$ can be represented by a matrix $\left(\begin{array}{cc}A & 0 \\ 0 & I\end{array}\right)$, where $A \in M_{2 n}(S)$ has only one non-zero entry in each row and column and this non-zero entry is either $1, \alpha$, or $\alpha^{-1}$. Since there are only a finite number of such matrices, $\left\langle\sigma_{1}, \cdots, \sigma_{n}\right\rangle$ is finite.

$R G$ is regular. This follows from Proposition 1.1.

$R^{G}$ is not regular. Indeed, if $x \in R^{G}$, then there exists $n \in \mathbb{N}$ such that $x_{n}=x_{n+h}$ for every $h \in \mathbb{N}$. let $k \in \mathbb{N}$ be such that $n+1 \leq 2 k$; then $x_{n}=x_{n+1}=\alpha\left(x_{n}\right)$ or $x_{n}=x_{n+1}=\alpha^{-1}\left(x_{n}\right)$ and hence $x_{n}=\left(\begin{array}{ll}a & 0 \\ b & a\end{array}\right)$ for some $a, b \in K$. But in this case $x_{i}=\left(\begin{array}{ll}a & 0 \\ b & a\end{array}\right)$ for every $i \in \mathbb{N}$. Thus $R^{G}$ is isomorphic to $\left\{\left(\begin{array}{ll}a & 0 \\ b & a\end{array}\right) \mid a, b \in K\right\}$ which is not regular. 
The necessary conditions of Theorem 2.4 turn out to be sufficient when the action is $G$-Galois. The action is said to be $G$-Galois if $R_{R^{G}}$ is finitely generated projective and the canonical homomorphism $R G \rightarrow$ End $\left(R_{R^{G}}\right)$ is a ring isomorphism. This is equivalent to $G$ being finite and $R \pi R=R G$. (See $[6,16]$.) If $R$ is simple, $G$ is finite, and the action is outer, then $R G$ is simple and consequently the action is $G$-Galois.

PROPOSITION 2.8. Let $R$ be a ring with $G$-Galois action. $R G$ is regular if and only if $R^{G}$ is regular. Furthermore, in this case $R G$ and $R^{G}$ are Morita equivalent.

PROOF. We already know that if $R G$ is regular, then $R^{G}$ is regular. Assume $R^{G}$ is regular; then $R G \cong$ End $\left(R_{R^{G}}\right)$ is also regular [8, Theorem 1.7]. In this case $1 \in \operatorname{tr}(R)$, hence $R_{R^{G}}$ is a progenerator and therefore $R G$ and $R^{G}$ are Morita equivalent.

REMARK. Unfortunately Proposition 2.8 cannot be generalized to an arbitrary group action. Indeed, let $K$ be a field of characteristic 2 and let $R=M_{2}(K)$. Consider $G$ to be the group of invertible matrices in $M_{2}\left(\mathbb{Z}_{2}\right)$ acting on $R$ by conjugation. It is easy to verify that $R^{G}=\left\{\left(\begin{array}{ll}a & 0 \\ 0 & a\end{array}\right) \mid a \in K\right\}$ and $\operatorname{tr}\left(\left(\begin{array}{ll}1 & 0 \\ 0 & 0\end{array}\right)\right)=\left(\begin{array}{ll}1 & 0 \\ 0 & 1\end{array}\right)$. If $R G$ is regular then $R H$ is also regular, where $H$ is the subgroup generated by $\left(\begin{array}{ll}1 & 1 \\ 0 & 1\end{array}\right)$. Hence $R^{H}$ is also regular; but this is not the case because $R^{H}=\left\{\left(\begin{array}{cc}a & b \\ 0 & a\end{array}\right) \mid a, b \in K\right\}$. Therefore $R G$ is not regular.

We finish this section with another special case where we have a complete characterization of regular skew group rings.

PROPOSITION 2.9. Assume that any non-trivial element of $G$ has order 2 and that $R$ is an algebra over a field $K$. Then $R G$ is regular if and only if $R^{H}$ is regular and $1 \in \operatorname{tr}_{H}(R)$ for every finite subgroup $H$ of $G$.

PROOF. We already know that the necessary conditions hold. In the other direction, from the assumption on $G$, we know that $G$ is abelian and locally finite. Therefore, in order to prove that $R G$ is regular, it is enough to assume that $G$ is finite and isomorphic to a finite direct product of copies of the group with two elements. Moreover, if $K$ has characteristic different from 2, then the claim follows from Proposition 1.1.

Assume $K$ has characteristic 2. We will finish the proof by induction on the order of $G$. Assume $|G|=2$ and let $r \in R$ with $\operatorname{tr}(R)=1$; then $r \pi+\pi\left({ }^{g} r\right)=1$ and hence the action is $G$-Galois. The regularity of $R G$ follows from Proposition 2.8. Now assume that $2<|G|$. There exist two subgroups $H$ and $L$ with $|H|=2$ and $G=H \times L$. Then $H$ acts on $R L$ by ${ }^{h} \alpha=h \alpha h^{-1}$. By the induction hypothesis $R L$ is regular and 
obviously $1 \in \operatorname{tr}_{H}(R L)$. Moreover, $R G=(R L) H$. Therefore it only remains to show that $(R L)^{H}$ is regular. Note that $R^{H}$ is $L$-invariant and $(R L)^{H}=\left(R^{H}\right) L$, furthermore for every subgroup $L^{\prime}$ of $L,\left(R^{H}\right)^{L^{\prime}}=R^{H \times L^{\prime}}$ is regular, and if $\operatorname{tr}_{H \times L^{\prime}}(r)=1$, then $\operatorname{tr}_{L^{\prime}}\left(\operatorname{tr}_{H}(r)\right)=\operatorname{tr}_{H \times L^{\prime}}(r)=1$ and $\operatorname{tr}_{H}(r) \in R^{H}$. Therefore $\left(R^{H}\right) L$ is regular by the induction hypothesis.

\section{Abelian regular rings}

In this section we present another case in which the necessary conditions are also sufficient.

Let $R$ be an abelian regular ring as in [8] (that is, $R$ is a regular ring and all idempotents are central). Let $G$ be a finite group acting on $R$ and let $B=B(R)$ be the boolean algebra of idempotents of $R$, with operations $e \vee f=e+f-e f$ and $e \wedge f=e f$. Clearly $\sigma_{g}(e \vee f)=\sigma_{g}(e) \vee \sigma_{g}(f)$ and $\sigma_{g}(e \wedge f)=\sigma_{g}(e) \wedge \sigma_{g}(f)$; therefore $G$ acts on $B$ and $B^{G}=B\left(R^{G}\right)$. Since $B^{G} \subseteq Z(R G)$, for every prime ideal $m$ of $B^{G}$ we can consider the central localizations $(R G)_{m}=(R G)_{B^{G} \backslash m}$ and $R_{m}$. Obviously $R_{m}$ is contained in $(R G)_{m}, R_{m}$ is $G$-invariant and $(R G)_{m} \cong R_{m} G$.

PROPOSITION 3.1. With the above conditions, $R_{m}$ is regular and 0 and 1 are the unique idempotents of $\left(R_{m}\right)^{G}$.

PROOF. Let $\phi_{m}: R \rightarrow R_{m}$ be the canonical map. If $e \in B^{G} \backslash m$ then $e(e-1)=0$ and hence $\phi_{m}(e)=\phi_{m}(1)=1$. Therefore $r / e=\phi_{m}(r) \phi_{m}(e)^{-1}=\phi_{m}(r)$ and hence $\phi_{m}$ is epic. We now show that $\operatorname{Ker}\left(\phi_{m}\right)=m R$. If $x \in m$, then $1-x \in B^{G} \backslash m$ and $(1-x) x=0$; thus $\phi_{m}(x)=0$. Now let $x \in \operatorname{Ker}\left(\phi_{m}\right)$. Then $e x=0$ for some $e \in B^{G} \backslash m$; and thus $x=(1-e) x$ and $1-e \in m$. Therefore $R_{m} \cong R / R m$. If $p$ is an idempotent of $R / R m$ which is fixed by the action of $G$, then there exists $e \in B$ such that $\bar{e}=p$. Since $p$ is fixed, $e$ can be chosen in $B^{G}$ (otherwise we can change it by $\left.f=\bigvee_{g \in G} \sigma_{g}(e)\right)$ and thus $e \in m$ or $1-e \in m$. But $p=\bar{e}$, so $p=0$ or $p=1$.

LEMMA 3.2. Let $G$ be a finite group acting on an abelian regular ring $R$ such that the only fixed idempotents are 0 and 1 . Then $R$ is semisimple artinian. In fact, $R \cong D^{n}$, where $D$ is a division ring and the action of $G$ on $R$ permutes transitively the factors of $D^{n}$.

PROOF. First we will see that $R$ has minimal idempotents. If not, for every nonzero idempotent $f \in R$ there are orthogonal non-zero idempotents $f_{1}$ and $f_{2}$ such that $f=f_{1}+f_{2}$. Now $\bigvee_{g \in G} \sigma_{g}\left(f_{1}\right)=1$. So there exists $x \in G$ such that $f_{2} \sigma_{x^{-1}}\left(f_{1}\right) \neq 0$, and then $g_{1}=f_{2} \sigma_{x^{-1}}\left(f_{1}\right)$ and $g_{2}=f_{1}$ are non-zero orthogonal idempotents such that 
$\sigma_{x}\left(g_{1}\right) \leq g_{2}$. We can construct in this way, by induction, two sequences of non-zero idempotents $\left\{f_{1, k}\right\}$ and $\left\{f_{2, k}\right\}$ such that for all $k \in \mathbb{N}, f_{1, k}$ and $f_{2, k}$ are orthogonal, $f_{1, k+1}<f_{1, k}$ and $f_{2, k+1}<f_{1, k}$ and $\sigma_{x_{k}}\left(f_{1, k}\right) \leq f_{2, k}$ for some $x_{k} \in G$. Observe that all $x_{k}$ must be different; thus since $G$ is finite we obtain a contradiction and there exists a minimal idempotent.

Let $e$ be a minimal idempotent of $R$. If $D=e R$, then $D$ is a division ring. Therefore, if $e_{1}, e_{2}, \ldots, e_{n}$ are the different elements of the form $\sigma_{g}(e)$ for some $g \in G$, we can express $R=e_{1} R \oplus e_{2} R \oplus \cdots \oplus e_{n} R \cong D^{n}$ and the action of $G$ permutes transitively the factors.

THEOREM 3.3. Let $R$ be an abelian regular ring and let $G$ be a finite group acting on $R$. If $1 \in \operatorname{tr}(R)$, then $R G$ is regular.

PROOF. Firstly we note that $(R G)_{m}$ is regular for any $m \in \operatorname{Spec}\left(B^{G}\right)$. This is because $(R G)_{m}=R_{m} G, R_{m}$ has no fixed idempotents other than 0 and 1 and then, by Lemma 3.2, $R_{m}$ and hence $R_{m} G$ is artinian. By [19, Theorem 27.7] $R_{m} G$ is also semiprime and hence it is regular.

Let $x \in R G$ and set $I=\left\{f \in B^{G} \mid\right.$ there exists $y \in R G$ such that $\left.f x=x y x\right\}$. Clearly if $f \in I$, the corresponding element $y$ can be chosen in $f(R G)$. If $e \in B^{G}$ and $f \in I$, put $y_{1}=e y \in R G$; thus $e f x=x y_{1} x$. On the other hand, if $f_{1}, f_{2} \in I$, $f_{1} x=x y_{1} x$ and $f_{2} x=x y_{2} x$ by taking $y=y_{1}+\left(1-f_{1}\right) y_{2}$ one has:

$$
\left(f_{1} \vee f_{2}\right) x=\left(f_{1}+f_{2}-f_{1} f_{2}\right) x=x y_{1} x+x\left(1-f_{1}\right) y_{2} x=x y x
$$

So $I$ is an ideal of $B^{G}$. It only remains to prove that $I=B^{G}$. If not, let $m$ be a maximal ideal of $B^{G}$ containing $I$. Since $(R G)_{m}$ is regular, there exists $y \in R G$ such that $\phi_{m}(x)=\phi_{m}(x) \phi_{m}(y) \phi_{m}(x)$. Thus there exists $e \in B^{G} \backslash m$ such that $e(x-x y x)=0$. So $e \in I \subseteq m$ which yields a contradiction.

We finish with an example showing that $R G$ regular does not imply that $1 \in$ $\operatorname{tr}(Z(R))$ in general.

EXAMPLE 3.4. Let $K=\mathbb{Z}_{2}(t)$ be the field of fractions of $\mathbb{Z}_{2}[t]$ and consider the automorphism $\sigma: K \rightarrow K$ for which $\sigma(t)=t+1$. Let $D$ be the classical ring of quotients of the skew polynomial ring $K[x, \sigma]$. The automorphism $\sigma$ extends to an automorphism $\sigma$ of $D$ by setting $\sigma(x)=x$. Consider the group $G=\langle\sigma\rangle$; since $\operatorname{tr}(t)=1$ and $D$ is abelian regular, $D G$ is regular but $\operatorname{tr}(Z(D))$ does not contain 1 .

\section{4. $G$-invariant pseudo-rank functions}

In this section we first obtain a characterization of the regular skew group rings $R G$, where $G$ is a finite group acting on a regular right self-injective ring $R$. Then we 
give a new necessary condition for certain skew group rings to be regular.

If $R$ is a regular ring and $N$ is a $G$-invariant pseudo-rank function on $R$, we can extend $N$ to $R G$ following techniques of $[10,1]$. We also can extend $G$ to an action on the $N$-completion $\bar{R}$ of $R$. Generalizing a result of Kado [10, Theorem 7], we show that $\overline{R G} \cong \bar{R} G$ where $\overline{R G}$ is the completion of $R G$ with respect to the extension of $N$. This implies that if $R G$ is regular, then so is $\bar{R} G$. Since $\bar{R}$ is regular and self-injective, this gives us a useful tool to prove that certain skew group rings are not regular. As an application we construct a certain skew group ring $R G$ which is not regular, where $R$ is a simple regular ring and $G$ is an outer group of automorphisms which has order 2 . An example of such a skew group ring was given in [9, Prop. 8.2], but it turns out that their example gives a regular skew group ring. If $R$ is a ring, $J(R)$ will denote the Jacobson radical of $R$.

PROPOSITION 4.1. Let $G$ be a finite group acting on a regular right self-injective ring $R$. The following conditions are equivalent:

(a) $R G$ is regular.

(b) $R G$ is semiprimitive.

(c) $R G$ is semiprime.

Proof. The proofs that (a) implies (b) implies (c) are well known. Assume $R G$ is semiprime. By [19, Theorem 4.2] $J(R G)^{|G|} \subseteq J(R) G=0$. Moreover, $R G$ is regular right self-injective (see [12]) and consequently $R G=R G / J(R G)$ is regular and right self-injective.

One can see, by using the techniques in [19, Section 18], that, for a regular right self-injective ring $R$ and a finite group $G$ acting on $R, R G$ is semiprime if and only if the centralizer $C_{R G}(R)$ of $R$ in $R G$ is semiprime; and the latter is a finite extension of $C=Z(R)$. If, in addition, $R$ is prime, then $C_{R G}(R)$ is isomorphic to $C^{\prime}\left[G_{\mathrm{inn}}\right]$ where $G_{\text {inn }}$ is the normal subgroup of $G$ consisting of elements that act by inner automorphisms, and $C^{t}\left[G_{\mathrm{inn}}\right]$ is some twisted group ring of $G_{\mathrm{inn}}$ over $C$. See [19, Lemma 12.4].

Let $R$ be any ring. A pseudo-rank function on $R$ is a map $N: R \rightarrow[0,1]$ such that:

(a) $N(x+y) \leq N(x)+N(y)$;

(b) $N(x y) \leq \min (N(x), N(y))$;

(c) $N(e+f)=N(e)+N(f)$ for any two orthogonal idempotents $e, f \in R$;

(d) $N(1)=1$.

A rank function is a pseudo-rank function with the additional property:

(e) $N(x)=0$ if and only if $x=0$. 
When $R$ is a regular ring, Condition (a) follows from (b), (c) and (d). If $R$ is a ring with pseudo-rank function $N$ the rule $\delta(x, y)=N(x-y)$ defines a pseudo-metric on $R$, which is a metric if and only if $N$ is a rank function. Since the operations on $R$ are continuous in the pseudo-metric, the (Hausdorff) completion $\bar{R}$ of $R$ becomes a ring called the $N$-completion of $R$. If $R$ is regular then $\bar{R}$ is a unit-regular, left and right self-injective ring: see [8, Theorem 19.7]. Let $G$ be a group acting on a ring $R$. A pseudo-rank function $N$ on $R$ is called $G$-invariant if $N\left({ }^{g} r\right)=N(r)$ for all $r \in R$, $g \in G$. It is easy to prove that if the action of $G$ is inner or locally inner (in the sense that for every $x \in R, g \in G$ there exists a unit $u \in R$ such that ${ }^{8} x=u^{-1} x u$ ), then every pseudo-rank function on $R$ is $G$-invariant. If $N$ is a $G$-invariant pseudo-rank function on $R$, the action of $G$ can be extended to the completion $\bar{R}$ in the obvious way.

Denote by $P(R)$ the set of all pseudo-rank functions on a regular ring $R$. Then $P(R)$ is a compact convex subset of $\mathbb{B}^{R}$; moreover, there exists an affine homeomorphism $P(R) \cong S\left(K_{o}(R),[R]\right)$, where $S\left(K_{o}(R),[R]\right)$ is the state space of the partially pre-ordered abelian group $K_{o}(R)$ with order-unit $[R]$; see [8, Propositions 16.17 and 17.12].

If $G$ is a group acting on a ring $R$, the rule $N^{g}(x)=N\left({ }^{g} x\right)$ defines an action of $G$ on $P(R)$ by affine homeomorphisms. Similarly we can define an action of $G$ on $S\left(K_{o}(R),[R]\right)$ such that the canonical affine homeomorphism from $P(R)$ onto $S\left(K_{o}(R),[R]\right)$ is $G$-equivariant. If $G$ is finite we can see, as in [1, Theorem 3], that there exists an affine embedding from $S\left(K_{o}(R),[R]\right)^{G}$ into $S\left(K_{o}(R G),[R G]\right)$.

It is obvious that, if $R$ admits a unique pseudo-rank function $N$, then $N$ is $G$ invariant for any group $G$ acting on $R$. Note also that if $N$ is a pseudo-rank function on $R$, then $N_{o}=|G|^{-1} \sum_{g \in G} N^{8}$ is a $G$-invariant pseudo-rank function on $R$.

The following result was obtained by Kado [10] in the special case that $|G|^{-1} \in R$ and $N$ is an extreme point in $P(R)$. For a Cauchy sequence $\left\{x^{(k)}\right\}$ in $X,\left[x^{(k)}\right]$ will denote its equivalence class in the completion $\bar{X}$.

THEOREM 4.2. Let $G$ be a finite group acting on a regular ring $R$ and let $N$ be a $G$-invariant pseudo-rank function on $R$. Let $\bar{N}$ be the rank function obtained by extending $N$ to $\bar{R}$. Then:

(a) $N$ induces a pseudo-rank function $\tilde{N}$ on $R G$ and the $\tilde{N}$-completion of $R G$ is isomorphic to $\bar{R} G$.

(b) The rank function induced by $\bar{N}$ on $\bar{R} G$ corresponds with the natural extension of $\tilde{N}$ to $\overline{R G}$ under the isomorphism in (a).

Proof. As in $[10,1]$ we can define $\tilde{N}$ in the following way:

$$
\tilde{N}(x)=|G|^{-1} s\left(\left[x R G_{R}\right]\right), \quad x \in R G
$$


where $s$ is the state corresponding to the pseudo-rank function $N$ in the affine homeomorphism $P(R) \cong S\left(K_{o}(R),[R]\right)$. Set $n=|G|$ and note that $R G \cong R^{n}$ as right $R$-modules. We will see that the $\tilde{N}$-topology on $R G$ is the same as the product topology on $R^{n}$. In order to show this, we need the following:

Claim 1. $s\left(\left[\left(\sum_{g \in G} a_{g} g\right) R G_{R}\right]\right) \leq \sum_{g \in G} s\left(\left[a_{g} R G_{R}\right]\right)$ where $a_{g} \in R$ for all $g \in G$.

Claim 2. For all $a \in R, s\left(\left[a R G_{R}\right]\right)=|G| N(a)$.

Proof of claim 1: We have $\left(\sum_{g \in G} a_{g} g\right) R G \leq \sum_{g \in G}\left(a_{g} g\right) R G=\sum_{g \in G} a_{g} R G$ and $\sum_{g \in G}\left(a_{g} R G\right)_{R}$ is isomorphic to a direct summand of $\bigoplus_{g \in G}\left(a_{g} R G\right)_{R}$ since $\sum_{g \in G}\left(a_{g} R G\right)_{R}$ is projective. We conclude that $s\left(\left[\left(\sum_{g \in G} a_{g} g\right) R G_{R}\right]\right) \leq \sum_{g \in G} s\left(\left[a_{g} R G_{R}\right]\right)$. Proof of claim 2: Observe that $R G=\bigoplus_{g \in G} g R$ and obviously $g R_{R} \cong R_{R}$ by left multiplication by $g$. Now $a R G_{R}=\bigoplus_{g \in G} a g R=\bigoplus_{g \in G} g\left(g^{g^{-1}} a\right) R \cong \bigoplus_{g \in G}\left(g^{-1} a\right) R$. Consequently $s\left(\left[a R G_{R}\right]\right)=\sum_{g \in G} s\left(\left[\left(g^{-1} a\right) R\right]\right)=\sum_{g \in G} N\left(g^{-1} a\right)=|G| N(a)$ since $N$ is $G$-invariant.

To see that the isomorphism $R G \cong R^{n}$ is a homeomorphism, it suffices to show that for sequences $\left\{a_{g}^{(k)}\right\}_{k=1}^{\infty}$ for each $g \in G, \lim _{k \rightarrow \infty} \sum_{g \in G} a_{g}^{(k)} g=0$ in the $\tilde{N}$-metric if and only if $\lim _{k \rightarrow \infty} a_{g}^{(k)}=0$ in the $N$-metric for all $g \in G$.

Assume first that $\lim _{k \rightarrow \infty} a_{g}^{(k)}=0$ in the $N$-metric. By using Claims 1 and 2, we compute:

$$
\begin{aligned}
\tilde{N}\left(\sum_{g \in G} a_{g}^{(k)} g\right) & =|G|^{-1} s\left(\left[\left(\sum_{g \in G} a_{g}^{(k)} g\right) R G_{R}\right]\right) \\
& \leq|G|^{-1} \sum_{g \in G} s\left(\left[a_{g}^{(k)} R G_{R}\right]\right)=\sum_{g \in G} N\left(a_{g}^{(k)}\right)
\end{aligned}
$$

and it follows that $\tilde{N}\left(\sum_{g \in G} a_{g}^{(k)} g\right) \rightarrow 0$ as $k \rightarrow \infty$.

Now assume that $\lim _{k \rightarrow \infty} \sum_{g \in G} a_{g}^{(k)} g=0$ in the $\tilde{N}$-metric. Fix $h \in G$ and observe that $\left(\sum_{g \in G} a_{g}^{(k)} g\right) R \leq \bigoplus_{g \in G} a_{g}^{(k)} g R$. Consider the canonical projection $\bigoplus_{g \in G} a_{g}^{(k)} g R \rightarrow a_{h}^{(k)} h R$. The restriction of this map to $\left(\sum_{g \in G} a_{g}^{(k)} g\right) R$ gives us an onto homomorphism of $R$-modules $\left(\sum_{g \in G} a_{g}^{(k)} g\right) R \rightarrow a_{h}^{(k)} h R$. Since $a_{h}^{(k)} h R$ is a projective $R$-module, $a_{h}^{(k)} h R$ is isomorphic to a direct summand of $\left(\sum_{g \in G} a_{g}^{(k)} g\right) R$. Consequently:

$$
\begin{aligned}
\tilde{N}\left(\sum_{g \in G} a_{g}^{(k)} g\right) & =|G|^{-1} s\left(\left[\left(\sum_{g \in G} a_{g}^{(k)} g\right) R G_{R}\right]\right) \geq|G|^{-1} s\left(\left[\left(\sum_{g \in G} a_{g}^{(k)} g\right) R\right]\right) \\
& \geq|G|^{-1} s\left(\left[a_{h}^{(k)} h R\right]\right)=|G|^{-1} s\left(\left[h^{-1}\left(a_{h}^{(k)}\right) R\right]\right)=|G|^{-1} N\left(h^{-1}\left(a_{h}^{(k)}\right)\right) \\
& =|G|^{-1} N\left(a_{h}^{(k)}\right) .
\end{aligned}
$$

It follows that $\lim _{k \rightarrow \infty} N\left(a_{h}^{(k)}\right)=0$. Since this holds for every $h \in G$, we have proved our statement about the topologies. 
In particular we see that a sequence $\left\{\sum_{g \in G} a_{g}^{(k)} g\right\}$ is a Cauchy sequence in the $\tilde{N}$-topology if and only if $\left\{a_{g}^{(k)}\right\}$ is a Cauchy sequence in the $N$-topology for all $g \in G$. Consequently the map $\overline{R G} \rightarrow \bar{R} G$ which sends $\left[\sum_{g \in G} a_{g}^{(k)} g\right]$ to $\sum_{g \in G}\left[a_{g}^{(k)}\right] g$, is well defined and a ring isomorphism. It is now straightforward to see that the natural extension of $\tilde{N}$ to $\overline{R G}$ corresponds to the extension of the ( $G$-invariant) rank function $\bar{N}$ to $\bar{R} G$ under the isomorphism defined above.

COROLLARY 4.3. Let $G$ be a finite group acting on a regular ring $R$ and $N$ be a $G$-invariant pseudo-rank function on $R$.

(a) If $R G$ is regular, then $\bar{R} G$ is regular.

(b) If $\bar{R} G$ is regular, then $J(R G) \subseteq \operatorname{Ker}(\tilde{N})$. If, in addition, $N$ is a rank function then $R G$ is semiprimitive.

PROOF. (a) If $R G$ is regular, then so is its completion with respect to a pseudo-rank function. By Theorem 4.2 we have $\overline{R G} \cong \bar{R} G$, so we deduce that $\bar{R} G$ is regular. (b) By [19, Theorem 4.2] we have $J(R G)^{n}=0$ where $n=|G|$. Let $x \in J(R G)$. There exists a sequence $\left\{y_{k}\right\}$ in $R G$ such that the limit of $\left\{\tilde{N}\left(x-x y_{k} x\right)\right\}$ is 0 . Now we have $\tilde{N}(x)=\tilde{N}\left(x-\left(x y_{k}\right)^{n} x\right) \leq n \tilde{N}\left(x-x y_{k} x\right)$. It follows that $x \in \operatorname{Ker}(\tilde{N})$ and consequently $J(R G) \subseteq \operatorname{Ker}(\tilde{N})$.

We shall use Corollary 4.3 to obtain an example of a simple regular ring with an outer action such that the skew group ring is not regular. Such an example was offered in [9, Prop. 8.2], but in fact it gives a regular ring as we now show:

The correct form of the fixed ring should be

$$
A^{\sigma} \cap M_{2^{n+1}}(F)=\left\{\left(\begin{array}{cc}
P & Q \\
\operatorname{tr}(P) & P+\operatorname{tr}(P+Q)
\end{array}\right) \mid P, Q \in M_{2^{n}}(F)\right\}
$$

and if $[\alpha] \in A^{\sigma}$ with $P \in A_{n}=M_{2^{n}}(F)$ such that $[\alpha][P][\alpha]=[\alpha]$, then it is easy to see that $[\alpha]\left[\left(\begin{array}{cc}P & 0 \\ \operatorname{tr}(P) & P+\operatorname{tr}(P)\end{array}\right)\right][\alpha]=[\alpha]$ and the fixed ring is regular. Furthermore since the action is $G$-Galois, the skew group ring is also regular.

For a non-regular skew group ring we give the following example, which is a variation of the one given in [9]:

EXAMPLE 4.4. A simple regular ring with an outer action such that the skew group ring is not regular:

Let $F$ be the field with two elements. For any $n \geq 1$ let $R_{n}=M_{t(n)}(F)$ where $t(1)=1$ and $t(n+1)=2^{n} t(n)$. For any $n \geq 1$ consider the diagonal map $\psi_{n}: R_{n} \rightarrow$ $R_{n+1}$ and the canonical map $\phi_{n}: R_{n} \rightarrow R=\lim _{n \geq 1} M_{t(n)}(F)$. Clearly $R$ is a simple regular ring. 
For any $n \geq 1$ define $\lambda_{n} \in R_{n}$ by $\lambda_{1}=1$ and $\lambda_{n}=\left(\begin{array}{ccccc}\mathrm{I} & 0 & \ldots & 0 & \mathrm{I} \\ 0 & \mathrm{I} & \ldots & 0 & 0 \\ . & . & \ldots & . & . \\ 0 & 0 & \ldots & 0 & \mathrm{I}\end{array}\right)$ where the matrix is decomposed into $t(n-1)$-blocks in $R_{n}$ and $I$ denotes the identity matrix. Define $x_{n}$ by induction as follows: $x_{1}=1 \in R_{1}$ and

$$
x_{n+1}=\left(\begin{array}{ccccc}
x_{n} & 0 & \ldots & 0 & \lambda_{n} x_{n} \\
0 & x_{n} & \ldots & 0 & 0 \\
. & . & \ldots & . & . \\
0 & 0 & \ldots & 0 & x_{n}
\end{array}\right) \in R_{n+1}
$$

It is easy to see that $\lambda_{n}$ commutes with $x_{n}$ and that $x_{n}^{2}=1$ for all $n \geq 1$. Now define an automorphism $\sigma$ of $R$ as follows:

$$
\sigma\left(\phi_{n}(A)\right)=\phi_{n+1}\left(x_{n+1} \cdot \psi_{n}(A) \cdot x_{n+1}\right) \quad\left(A \in R_{n}\right)
$$

Since $\lambda_{n+1} \in C_{R_{n+1}}\left(\psi_{n}\left(R_{n}\right)\right)$, it is easy to see that $\sigma$ is a well defined automorphism of order two on $R$, and clearly $\sigma$ is outer; furthermore let $G=\langle\sigma\rangle$.

Let $N$ be the unique rank function on $R$, given by $N\left(\phi_{n}(A)\right)=\operatorname{rank}(A) / t(n)$ for $A \in R_{n}$. The rank function $N$ is $G$-invariant and $\sigma$ extends to an automorphism $\bar{\sigma}$ of $\bar{R}$. Since $N\left(\phi_{n+1}\left(x_{n+1}\right)-\phi_{n}\left(x_{n}\right)\right)=1 / 2^{n}$, we see that $\left\{\phi_{n}\left(x_{n}\right)\right\}$ is a Cauchy sequence in $R$. Now put $x=\lim _{n \rightarrow \infty} \phi_{n}\left(x_{n}\right) \in \bar{R}$. It is clear that $\bar{\sigma}$ is an inner automorphism of $\bar{R}$, given by conjugation by $x$. Since the center of $\bar{R}$ is isomorphic to $F$ (see [7, Theorem 2.8(c)]), and $G_{\text {inn }}=G$ on $\bar{R}$, we obtain that $C^{t}\left[G_{\text {inn }}\right]=F[G]$ is not semiprime. It follows from [19, Lemma $18.8(\mathrm{ii})]$ that $\bar{R} G$ is not semiprime and consequently not regular. Finally, Corollary 4.3 (a) implies that $R G$ is not regular.

REMARK. Note that in the previous example $R^{G}$ is not regular and $1 \in \operatorname{tr}(R)$. Indeed, the first is a consequence of Proposition 2.8, and an element of trace 1 is $\phi_{3}(\omega)$ where $\omega$, decomposed in 2-blocks, is given by

$$
\omega=\left(\begin{array}{llll}
x & 0 & 0 & y \\
0 & x & 0 & 0 \\
0 & 0 & x & 0 \\
0 & 0 & 0 & x
\end{array}\right) \quad \text { where } \quad x=\left(\begin{array}{ll}
1 & 0 \\
1 & 0
\end{array}\right), \quad y=\left(\begin{array}{ll}
0 & 0 \\
1 & 0
\end{array}\right)
$$

It follows that $R G$ and $R^{G}$ are Morita equivalent. In particular $R^{G}$ is a simple ring and its completion with respect to the rank function $N$ restricted to $R^{G}$ is $\bar{R}^{G}$ which is a self-injective non-regular ring.

Finally we obtain a result which implies that in Example 4.4 the ring $R G$ is neither left nor right P. P. (A ring $R$ is said to be left $P$. $P$. if every cyclic left ideal is projective.) 
PROPOSITION 4.5. Let $G$ be a finite group acting on a regular ring $R$. If $R G$ is a left P.P. ring, then $R^{G}$ is regular.

PROOF. Let $r \in R^{G}$. Since $P=(R G)(\pi r)$ is a projective $R G$-module, the map $R G \rightarrow P$ given by right multiplication by $\pi r$ splits. Let $\varphi: P \rightarrow R G$ be a splitting for this map. Write $\varphi(\pi r)=\sum_{g \in G} a_{g} g$. As in the proof of Theorem 2.4, $a_{g}={ }^{g} a_{1}$ for all $g \in G$. Set $a=a_{1}$. Thus $a \in r_{R}\left(l_{R}(r)\right)=r R$ and consequently $a=r b$ for some $b \in R$. Note that $\pi r=\varphi(\pi r) \pi r=\pi a \cdot \pi r=\pi \operatorname{tr}(a) r$. So $r=\operatorname{tr}(a) r=\operatorname{tr}(r b) r=r \operatorname{tr}(b) r$. It follows that $R^{G}$ is regular.

It was claimed in [15, Corollary 4] that, for a simple ring $S$ and a finite group $G$ of outer automorphisms of $S, S^{G}$ is a simple ring if and only if the homological dimension of any left $S G$-module $A$ coincides with the homological dimension of $A$ viewed as an $S$-module. However, the ring $R$ of Example 4.4 gives a counterexample to this claim. Indeed, every cyclic left ideal of $R G$ is projective as a left $R$-module because $R$ is regular, but it follows from Proposition 4.5 that at least one cyclic left ideal of $R G$ is not projective as an $R G$-module.

\section{References}

[1] R. Alfaro, 'States on skew group rings and fixed rings', Comm. Algebra 18 (1990), 3381-3394.

[2] M. Auslander, 'On regular group rings', Proc. Amer. Math. Soc. 8 (1957), 658-664.

[3] M. Beattie, 'A generalization of the smash product of a graded ring', J. Pure Appl. Algebra 52 (1988), 219-226.

[4] M. Cohen and S. Montgomery, 'Group-graded rings, smash products and group actions', Trans. Amer. Math. Soc. 282 (1984), 237-258.

[5] I. G. Connell, 'On the group ring', Canad. J. Math. 15 (1963), 650-685.

[6] F. DeMeyer, 'Some notes on the general Galois theory of rings', Osaka J. Math. 2 (1965), 117-127.

[7] K. R. Goodearl, 'Centers of regular self-injective rings', Pacific J. Math. 76 (1978), 381-395.

[8] _- Von Neumann regular rings (Pitman, London, 1979).

[9] J. M. Goursaud, J. Osterburg, J. L. Pascaud and J. Valette, 'Points fixes des anneaux reguliers auto-injectifs à gauche', Comm. Algebra 9 (1981), 1343-1394.

[10] J. Kado, 'Pseudo-rank functions on crossed products of finite groups over regular rings', Osaka J. Math. 22 (1985), 821-833.

[11] S. Montgomery, Fixed rings of finite automorphism groups of associative rings, volume 818 of Lecture Notes in Mathematics (Springer, Berlin, 1980).

[12] C. Năstăsescu, 'Group rings of graded rings, applications', J. Pure Appl. Algebra 33 (1984), 315-335.

[13] C. Naståsescu, M. Van. der Bergh and F. Van Oystaeyen, 'Seperable functors, applications to graded rings and modules', J. Algebra 123 (1989), 397-413.

[14] C. Năstăsescu and F. Van Oystaeyen, Graded ring theory (North-Holland, Amsterdam, 1982).

[15] J. Osterburg, 'Fixed rings of simple rings', Comm. Algebra 6 (1978), 1741-1750.

[16] -, 'Smash products and G-Galois actions', Proc. Amer. Math. Soc. 98 (1986), 217-221. 
[17] A. Page, 'Action de groupe', Seminaire d'Algebra P. Dubreil, volume 740 of Lecture Notes in Mathematics (Springer, Berlin, 1979).

[18] D. S. Passman, The algebraic structure of group rings (Wiley, New York, 1977).

[19] -, Infinite crossed products, volume 135 of Pure and Applied Mathematics (Academic Press, New York, 1989).

[20] M. D. Rafael, 'Separable functors revisited', Comm. Algebra 18 (1990), 1445-1549.

[21] O. E. Villamayor, 'On weak dimension of algebras', Pacific J. Math. 9 (1959), 941-951.

Department of Mathematics

University of Michigan-Flint

Flint, MI 48502

U.S.A.

Departamento de Matemáticas

Universidad de Murcia

(30001) Murcia

Spain
Departament de Matemàtiques

Universitat Autònoma de Barcelona (08193) Bellaterra (Barcelona)

Spain 